\title{
Quality of life of lung cancer patients receiving outpatient chemotherapy
}

\author{
AYAKO MATSUDA ${ }^{1}$, MIKA KOBAYASHI $^{1}$, YUMI SAKAKIBARA ${ }^{2}$, MEIYO TAMAOKA $^{2}$, \\ MASASHI FURUIYE ${ }^{2}$, NAOHIKO INASE ${ }^{2}$ and EISUKE MATSUSHIMA ${ }^{1}$ \\ Departments of ${ }^{1}$ Liaison Psychiatry and Palliative Medicine, and ${ }^{2}$ Integrated Pulmonology, \\ Graduate School of Medical and Dental Sciences, Tokyo Medical and Dental University, Tokyo, Japan
}

Received October 26, 2010; Accepted December 27, 2010

DOI: $10.3892 /$ etm.2011.185

\begin{abstract}
An increasing number of cancer patients receive outpatient chemotherapy as an alternative to inpatient chemotherapy. The aim of this study was to investigate whether quality of life (QOL) during outpatient chemotherapy was better than QOL prior to hospital discharge, and to explore possible related factors prior to hospital discharge that affected the QOL of lung cancer patients who received outpatient chemotherapy. Lung cancer inpatients who were scheduled for outpatient chemotherapy were assessed two times (prior to hospital discharge and during outpatient chemotherapy) using the Functional Assessment of Cancer Therapy-Lung and Hospital Anxiety and Depression Scale. A total of 40 patients completed all assessments, both prior to hospital discharge and during outpatient chemotherapy. In the present study, QOL during outpatient chemotherapy was not significantly different when compared with the QOL prior to hospital discharge, and predictors prior to hospital discharge for a better QOL of patients during outpatient chemotherapy included better social, emotional and physical well-being. These results suggest that medical staff, in particular those involved in outpatient chemotherapy, need to recognize social and emotional as well as physical well-being prior to hospital discharge, regardless of cancer-related factors and the personal characteristics of the patients.
\end{abstract}

\section{Introduction}

Lung cancer is the most common cancer worldwide, both in terms of incidence and mortality (1). In Japan, lung cancer is the second most common cancer and the most prevalent cancer implicated in mortality (2). Small-cell lung cancer (SCLC) accounts for approximately $20 \%$ (3) and non-small cell lung

Correspondence to: Ayako Matsuda, Department of Liaison Psychiatry and Palliative Medicine, Graduate School of Medical and Dental Sciences, Tokyo Medical and Dental University, 1-5-45 Yushima, Bunkyo-ku, Tokyo 113-8519, Japan

E-mail: ayaklppm@tmd.ac.jp

Key words: chemotherapy, lung cancer, outpatient, quality of life cancer (NSCLC) accounts for approximately 80\% of lung cancers (4). For SCLC, treatment is generally aggressive and primarily based on chemotherapy. For the treatment of NSCLC, surgery is the first choice in the early stages, a combination of chemotherapy and radiotherapy is the first choice for locally advanced disease, and chemotherapy alone is presently the best therapeutic option for metastatic patients (5). For NSCLC patients with early stage disease who undergo surgery, adjuvant chemotherapy has become the standard of care (6). The benefit of chemotherapy in this disease is modest, but novel drugs and combined strategies may offer improved survival rates (4). In patients with advanced NSCLC and extensive SCLC, the first choice in treatment is palliative chemotherapy, aiming to prolong overall survival, alleviate disease-related symptoms and improve quality of life (QOL) $(7,8)$.

An increasing number of cancer patients receive outpatient chemotherapy as an alternative to inpatient chemotherapy in the cases of SCLC or unresectable NSCLC. Postoperative adjuvant chemotherapy for NSCLC is an option for suitable cases of NSCLC. Patient suitability for outpatient chemotherapy depends on having a favorable performance status and a nearby residence (9). Patients who are scheduled for outpatient chemotherapy usually first receive one or two cycles of chemotherapy as inpatients. Previous studies have argued that the QOL of lung cancer patients is maintained and even improves during outpatient chemotherapy $(9,10)$. The American Society of Clinical Oncology has described that cancer-related QOL is crucial since it is the patients' evaluation of how their cancer and its treatment have affected the physical, psychological and social aspects of their lives (11). Gridelli et al reported that improving the understanding of the impact of available treatments on patient QOL is mandatory, and this understanding can be addressed only through well-planned and well-conducted prospective clinical trials (5). However, it is challenging for medical staff to precisely measure the QOL of outpatients receiving chemotherapy compared to inpatients due to insufficient staff and systems.

A review of the literature on QOL in patients with all types of lung cancer concluded that future challenges in lung cancer treatment should not only improve survival, but also, and perhaps more importantly, improve the QOL of patients (12). However, previous studies that have compared chemotherapy regimens and the QOL of lung cancer patients were 
restricted to defined samples of SCLC or NSCLC patients. Accordingly, few studies focused on the QOL of patients who received outpatient chemotherapy, including patients with SCLC, unresectable NSCLC and postoperative NSCLC. The present study was designed to survey outpatients receiving chemotherapy, including patients with SCLC, unresectable NSCLC and postoperative NSCLC.

The aim of this study was to investigate whether QOL during outpatient chemotherapy was better than that prior to hospital discharge and to explore related factors prior to hospital discharge that affected the QOL of lung cancer patients who received outpatient chemotherapy.

\section{Patients and methods}

Patients. Between June 2008 and June 2010, lung cancer inpatients at Tokyo Medical and Dental University Hospital who were scheduled for outpatient chemotherapy were consecutively recruited. The inclusion criteria included: age $\geq 20$ years, awareness of lung cancer diagnosis, ability to complete the questionnaire and participate in the study, no severe dementia, no psychiatric problems, no previous administration of outpatient chemotherapy and ability to provide informed consent.

Prior to participation, the patients provided written informed consent. The Tokyo Medical and Dental University Hospital Research Review Board approved the study and granted formal access to the patients.

Procedures. The patients were assessed with both the Functional Assessment of Cancer Therapy-Lung (FACT-L) and Hospital Anxiety and Depression Scale (HADS) two times. The two assessment points were prior to hospital discharge (baseline) and the median cycle of chemotherapy during outpatient chemotherapy. Of the patients who were included in the study, those who were able to complete all assessments both prior to hospital discharge and during outpatient chemotherapy were analyzed.

Questionnaires. FACT-L has been reported to be a reliable and valid measure (13). The version 4 of FACT-L is composed of the Functional Assessment of Cancer Therapy-General (FACT-G) and the Lung Cancer Subscale (LCS). The FACT-G consists of 27 items that measure physical well-being (PWB; 7 items), social/family well-being (SWB: 7 items), emotional well-being (EWB; 6 items) and functional well-being (FWB; 7 items). The LCS of the questionnaire consists of 7 items that measure symptoms specific to lung cancer. A higher score indicates a better QOL. HADS is a reliable and valid measure $(14,15)$. It is a self-rating type questionnaire with 14 items that measure anxiety (HADS-A) and depression (HADS-D). For these questionnaires, a higher score indicates a higher severity of symptoms. The cut-off point for HADS total is 11 points, and a score $\geq 11$ indicates depression (15).

Statistical analyses. The data were analyzed using the IBM SPSS Statistics 18. Initially, comparison of scores prior to hospital discharge and during outpatient chemotherapy for FACT-G and the subscale of FACT-L (PWB, SWB, EWB, FWB and LCS) was assessed using the Wilcoxon test. Multiple regression analysis was then used with stepwise variable
Table I. Patient characteristics.

No. of patients (\%)

\begin{tabular}{lr}
\hline No. of patients & \multicolumn{1}{l}{} \\
Mean age (SD) & $65.8(8.9)$ \\
Gender & \\
Male & $34(85.0)$ \\
Female & $6(15.0)$ \\
Married & \\
Yes & $29(72.5)$ \\
No & $11(27.5)$ \\
Cohabitation & \\
Yes & $33(82.5)$ \\
No & $7(17.5)$ \\
Occupation & \\
Yes & $13(32.5)$ \\
No & $27(67.5)$ \\
Smoking history & \\
Yes & $37(92.5)$ \\
No & $3(7.5)$ \\
Cell type & \\
Small-cell & $5(12.5)$ \\
Non-small cell & $35(87.5)$ \\
Cancer stage & \\
I/II & $7(17.5)$ \\
III/IV & $33(82.5)$ \\
Treatment & \\
Surgery + chemotherapy & $11(27.5)$ \\
Chemotherapy & $29(72.5)$ \\
HADS total, mean (SD) & \\
Prior to hospital discharge & $5.5(3.6)$ \\
Outpatient chemotherapy & $5.9(4.3)$ \\
\hline
\end{tabular}

SD, standard deviation; HADS, Hospital Anxiety and Depression Scale.

selection to test for factors that predicted FACT-G score during outpatient chemotherapy. The cancer-related variables (treatment, stage and cell type), personal characteristics (age, gender, marital status, cohabitation, occupation and smoking history), the FACT-L subscales (PWB, SWB, EWB, FWB and LCS) and HADS total score prior to hospital discharge were predefined as factors. The P-values reported are two-sided. A P-value of $<0.05$ was assumed to indicate statistical significance.

\section{Results}

Patients. Between June 2008 and June 2010, a total of 61 patients who met the study criteria were considered: 2 patients were excluded and 59 patients agreed to participate in the study. The reasons for exclusion were as follows: 1 patient refused to participate in the study and 1 patient was not assessed prior to hospital discharge. Of the 59 patients, 19 patients were not assessed during outpatient chemotherapy 
Table II. QOL of patients during outpatient chemotherapy in comparison to QOL prior to hospital discharge.

\begin{tabular}{|c|c|c|c|c|c|}
\hline \multirow[t]{2}{*}{ Scale } & \multicolumn{2}{|c|}{ Prior to hospital discharge $(n=40)$} & \multicolumn{2}{|c|}{ Outpatient chemotherapy $(n=40)$} & \multirow[t]{2}{*}{ P-value (Wilcoxon test) } \\
\hline & Median & IQR & Median & IQR & \\
\hline FACT-G & 71.9 & $66.4-78.0$ & 72.8 & $64.6-78.7$ & 0.742 \\
\hline PWB & 22.0 & $18.5-25.0$ & 22.0 & $19.0-24.0$ & 0.742 \\
\hline SWB & 17.5 & $11.4-21.0$ & 17.5 & $12.0-19.5$ & 0.209 \\
\hline EWB & 17.0 & $13.3-19.8$ & 19.0 & $14.0-21.0$ & 0.054 \\
\hline FWB & 17.5 & $14.0-20.0$ & 17.3 & $14.3-20.0$ & 0.627 \\
\hline LCS & 21.0 & $17.3-23.0$ & 20.0 & $17.3-22.0$ & 0.525 \\
\hline
\end{tabular}

QOL, quality of life; IQR, interquartile range; FACT-G, Functional Assessment of Cancer Therapy-General; PWB, physical well-being; SWB, social/family well-being; EWB, emotional well-being; FWB, functional well-being; LCS, lung cancer subscale.

Table III. Coefficients of multiple stepwise regression model for QOL variables prior to hospital discharge associated with FACT-G during outpatient chemotherapy.

\begin{tabular}{|c|c|c|c|c|}
\hline \multirow[t]{2}{*}{ Variable } & \multicolumn{2}{|c|}{ Unstandardized coefficient } & \multirow{2}{*}{$\frac{\text { Standardized coefficient }}{\beta}$} & \multirow[t]{2}{*}{ P-value } \\
\hline & $\beta$ & SE & & \\
\hline SWB & 1.12 & 0.22 & 0.62 & $<0.001$ \\
\hline EWB & 1.26 & 0.40 & 0.40 & 0.003 \\
\hline PWB & 1.34 & 0.37 & 0.41 & 0.001 \\
\hline
\end{tabular}

QOL, quality of life; FACT-G, Functional Assessment of Cancer Therapy-General; SWB, social/family well-being; EWB, emotional wellbeing; PWB, physical well-being; SE, standard error.

and 40 patients completed all assessments both prior to hospital discharge and during outpatient chemotherapy. The reasons why the 19 patients were not evaluated during outpatient chemotherapy were as follows: 17 patients were not able to receive outpatient chemotherapy and 2 patients were not met during their outpatient chemotherapy. Therefore, 40 patients were eligible as evaluable subjects and included in the analysis. The characteristics of the patients are summarized in Table I.

Comparison of quality of life scales. A comparison of the FACT-G total score $(\mathrm{P}=0.724)$, PWB $(\mathrm{P}=0.724)$, SWB $(\mathrm{P}=0.209)$, EWB $(\mathrm{P}=0.054), \mathrm{FWB} \quad(\mathrm{P}=0.627)$ and LCS $(\mathrm{P}=0.525)$ in $\mathrm{QOL}$ prior to hospital discharge and during outpatient chemotherapy did not reveal any significant differences. The results are summarized in Table II.

Factors that predicted quality of life during outpatient chemotherapy. There were three statistically significant predictors (SWB: $\beta=1.12, \mathrm{P}<0.001$; EWB: $\beta=1.26, \mathrm{P}=0.003$; PWB: $\beta=1.34, \mathrm{P}=0.001$ ) for the FACT-G score during outpatient chemotherapy (Table III). Higher scores of SWB, EWB and PWB prior to hospital discharge were associated with greater improvement in FACT-G during outpatient chemotherapy.

\section{Discussion}

Upon comparing QOL in lung cancer patients prior to hospital discharge and that during outpatient chemotherapy, the present study indicated that the QOL during outpatient chemotherapy did not significantly differ from the QOL prior to hospital discharge. However, the QOL during outpatient chemotherapy was expected to improve compared to that prior to hospital discharge. Patients receiving outpatient chemotherapy were able to go home after finishing the chemotherapy doses and live at their residence until their next visit to the doctor and dose of chemotherapy. Montazeri et al described that family and leisure time was as important as physical health for lung cancer patients (12). In the event that medical staff are involved in predictors of improvement in QOL during outpatient chemotherapy even prior to hospital discharge, the QOL of the patients may improve.

In the present study, the predictors prior to hospital discharge for better QOL of patients during outpatient chemotherapy included better social, emotional and physical well-being. Therefore, since social well-being prior to hospital discharge affects the QOL of patients during outpatient chemotherapy, medical staff must assess these factors, and consider whether patients need supportive care. Freedman 
and Zimmerman described that supportive care for patients with advanced lung cancer should involve consideration and discussion of the therapeutic options (16). In addition, better emotional well-being prior to hospital discharge, as measured by FACT-L, was strongly related to better QOL during outpatient chemotherapy. Previous studies have reported that lung cancer patients, particularly those with more severe symptoms or functional limitations, suffer mood disturbances (17) and experience depression or anxiety disorders $(18,19)$. However, psychological morbidity, as measured by HADS, was not associated with a better QOL during outpatient chemotherapy. It is assumed that few patients who received outpatient chemotherapy in the present study experienced anxiety or depression as the majority of the patients did not have severe symptoms or functional limitations. Therefore, medical staff should note not only a negative emotional state such as anxiety or depression, but also the comprehensive dimension of emotional functioning. Concerning physical well-being, Hollen et al reported that physical and functional states were crucial predictors of QOL in patients receiving chemotherapy (20). The present results similarly indicated that a better physical well-being prior to hospital discharge was strongly related to a better QOL during outpatient chemotherapy.

Gralla et al described that the incorporation of the assessment of QOL as part of daily oncology practice may enhance current treatment strategies for cancer patients (21). In addition, enhanced understanding of the common QOL of patients is able to improve the clinical care of patients (22). Moreover, even though research incorporating symptoms and QOL measures is challenging, it is an essential part of supportive care (16).

The present study found that it is crucial to incorporate, in daily practice, an evaluation of the QOL of lung cancer patients who are scheduled for outpatient chemotherapy. Medical staff involved in outpatient chemotherapy in particular, need to recognize the social and emotional wellbeing of patients as well as their physical well-being prior to hospital discharge, regardless of cancer-related factors and personal characteristics. For lung cancer patients receiving outpatient chemotherapy, awareness of the QOL improves both medical and psychological aspects even prior to hospital discharge.

\section{Acknowledgements}

This study was supported by a grant from the Ministry of Health, Labor and Welfare (no. 2211271). We would like to thank all participants and medical staff involved in this study. The study sponsors had no role in the study design, in the collection, analysis and interpretation of data, in the writing of the manuscript, or in the decision to submit the manuscript for publication.

\section{References}

1. Parkin DM: Global cancer statistics in the year 2000. Lancet Oncol 2: 533-543, 2001

2. Center for Cancer Control and Information Services, National Cancer Center, Japan: Data source of Vital Statistics Japan (Ministry of Health, Labour and Welfare) http://ganjoho.ncc. go.jp/professional/statistics/statistics.html. Accessed October 1, 2006 (In Japanese).

3. Clark R and Ihde DC: Small-cell lung cancer: treatment progress and prospects. Oncology 12: 647-658, 1998.

4. Novello S and Le Chevaliar T: Chemotherapy for non-small-cell lung cancer. Part 1. Early-stage disease. Oncology 17: 357-364, 2003.

5. Gridelli C, Perrone F, Nelli F, Ramponi S and De Marinis F: Quality of life in lung cancer patients. Ann Oncol 12: S21-S25, 2001.

6. Ettinger DS, Akerley W, Bepler G, et al: Non-small cell lung cancer: clinical practice guidelines in oncology. J Natl Compr Canc Netw 6: 228-269, 2008.

7. De Petris L, Crinò L, Scagliotti GV, et al: Treatment of advanced non-small cell lung cancer. Ann Oncol 17: ii36-ii41, 2006.

8. Agra Y, Pelayo M, Sacristan M, Sacristan A, Serra C and Bonfill X: Chemotherapy versus best supportive care for extensive small-cell lung cancer. Cochrane Database Syst Rev 7: CD001990, 2009.

9. Yoneda S: Outpatient chemotherapy for lung cancer. Gan To Kagaku Ryoho 34: 533-537, 2007 (In Japanese).

10. Ishiura Y, Yamamoto $\mathrm{H}$, Terasaki $\mathrm{Y}$, et al: Analysis of factors associated with quality of life for patients with non-small lung cancer receiving outpatient vinorelbine therapy as alternative inpatient therapy. Gan To Kagaku Ryoho 34: 1401-1404, 2007 (In Japanese).

11. American Society of Clinical Oncology: Outcomes of cancer treatment for technology assessment and cancer treatment guidelines. J Clin Oncol 14: 671-679, 1996.

12. Montazeri A, Gillis CR and McEwen J: Quality of life in patients with lung cancer. Chest 113: 467-481, 1998.

13. Cella DF, Bonomi AE, Lloyd SR, Tulsky DS, Kaplan E and Bonomi P: Reliability and validity of the Functional Assessment of Cancer Therapy-Lung (FACT-L) quality of life instrument. Lung Cancer 12: 199-220, 1995.

14. Zigmond AS and Snaith RP: The hospital anxiety and depression scale. Acta Psychiatr Scand 67: 361-370, 1983.

15. Kugaya A, Akechi T, Okuyama T, Okamura $\mathrm{H}$ and Uchitomi $\mathrm{Y}$ : Screening for psychological distress in Japanese cancer patients. Jpn J Clin Oncol 28: 333-338, 1998.

16. Freedman OC and Zimmermann C: The role of palliative care in the lung cancer patient: can we improve quality while limiting futile care? Curr Opin Pulm Med 15: 321-326, 2009.

17. McCorkle R and Quint-Benoliel J: Symptom distress, current concerns and mood disturbance after diagnosis of life-threatening disease. Soc Sci Med 17: 431-438, 1983.

18. Hopwood P and Stephens RJ: Depression in patients with lung cancer: Prevalence and risk factors derived from quality-of-life data. J Clin Oncol 18: 893-903, 2000.

19. Derogatis LR, Morrow GR, Fetting J, et al: The prevalence of psychiatric disorders among cancer patients. JAMA 249: 751-757, 1983.

20. Hollen PJ, Gralla RJ, Kris MG, et al: Measurement of quality of life in patients with lung cancer in multicenter trials of new therapies. Psychometric assessment of the Lung Cancer Symptom Scale. Cancer 73: 2087-2098, 1994.

21. Gralla RJ and Thatcher N: Quality-of-life assessment in advanced lung cancer: considerations for evaluation in patients receiving chemotherapy. Lung Cancer 46: S41-S47, 2004.

22. Mystakidou K, Tsilika E, Parpa E, Smyrnioti M and Vlahos L: Assessment of anxiety and depression in advanced cancer patients and their relationship with quality of life. Qual Life Res 14: 1825-1833, 2005. 